\title{
TRUDINGER-MOSER TYPE INEQUALITIES FOR WEIGHTED SOBOLEV SPACES INVOLVING FRACTIONAL DIMENSIONS
}

\author{
JOSÉ FRANCISCO DE OLIVEIRA AND JOÃO MARCOS DO Ó \\ (Communicated by Walter Craig)
}

\begin{abstract}
We derive sharp Trudinger-Moser inequalities for weighted Sobolev spaces and prove the existence of extremal functions. The inequalities we obtain here extend for fractional dimensions the classical results in the radial case. The main ingredient used in our arguments reveals a new proof of a result due to J. Moser for which we give an improved version.
\end{abstract}

\section{INTRODUCTION}

Let $\Omega \subset \mathbb{R}^{n}(n \geq 2)$ be a smooth domain. The classical Sobolev embedding theorem asserts that the embedding $W_{0}^{1, n}(\Omega) \hookrightarrow L^{q}(\Omega)$ for any $1 \leq q<\infty$ is continuous, but easy examples show that $W_{0}^{1, n}(\Omega) \not \subset L^{\infty}(\Omega)$ (cf. [2]). When $\Omega$ is a bounded domain, replacing the target Lebesgue space by an Orlicz space, N. Trudinger 22 proved that there exists $\mu>0$ such that $W_{0}^{1, n}(\Omega)$ is embedded in the Orlicz space $L_{\phi}(\Omega)$ determined by $\phi(t)=e^{\mu|t|^{n /(n-1)}}-1$. This result was sharpened by J. Moser [19], who found the best exponent $\mu$ and proved the following result:

$$
\sup _{u \in W_{0}^{1, n}(\Omega):\|\nabla u\|_{n}=1} \int_{\Omega} e^{\mu|u|^{\frac{n}{n-1}}} \mathrm{~d} x \quad\left\{\begin{array}{lll}
\leq C_{n}|\Omega| & \text { if } & \mu \leq \mu_{n}:=n \omega_{n-1}^{1 /(n-1)}, \\
=\infty & \text { if } \quad \mu>\mu_{n},
\end{array}\right.
$$

where $|\Omega|$ denotes the Lebesgue measure of a set $\Omega$ in $\mathbb{R}^{n}$ and $\omega_{n-1}$ is the measure of the unit sphere in $\mathbb{R}^{n}$. Estimates like (1) are now called Trudinger-Moser type inequalities. For related works and applications we refer the reader to [8, 9, 18 , and the references therein.

To prove (1), J. Moser used symmetrization; that is, to every function $u$ is associated a radially symmetric function $u^{*}$ such that the sublevel-sets of $u^{*}$ are balls with the same area as the corresponding sublevel-sets of $u$, i.e. $\mid\left\{x \in \mathbb{R}^{n}\right.$ : $\left.u^{*}(x)<d\right\}|=|\{x \in \Omega: u(x)<d\} \mid$. Then $u^{*}$ is a positive and non-increasing function defined on $B_{R}(0)$ with $\left|B_{R}\right|=|\Omega|$. By construction, one has the following property: Let $f \in C(\mathbb{R})$; then $\int_{B_{R}} f\left(u^{*}\right) \mathrm{d} x=\int_{\Omega} f(u) \mathrm{d} x$; furthermore, the wellknown Pólya-Szegö inequality asserts that

$$
\int_{B_{R}}\left|\nabla u^{*}\right|^{n} \mathrm{~d} x \leq \int_{\Omega}|\nabla u|^{n} \mathrm{~d} x
$$

Received by the editors December 29, 2011 and, in revised form, May 19, 2012 and September 8, 2012.

2010 Mathematics Subject Classification. Primary 35J62, 46E35, 26D10, 35B33.

This research was partially supported by the National Institute of Science and Technology of Mathematics INCT-Mat, CAPES-PROCAD, CNPq grant 307400/2009-3 and 141853/2012-3, and $\mathrm{MCT} / \mathrm{CNPq} / \mathrm{MEC} / \mathrm{CAPES}$ grant 552758/2011-6. 
From this we clearly deduce that

$$
\sup _{\|\nabla u\|_{n} \leq 1} \int_{\Omega} e^{\mu|u|^{\frac{n}{n-1}}} \mathrm{~d} x \leq \sup _{\left\|\nabla u^{*}\right\|_{n} \leq 1} \int_{B_{R}} e^{\mu\left|u^{*}\right|^{\frac{n}{n-1}}} \mathrm{~d} x,
$$

and hence it is sufficient to consider symmetric non-negative and non-increasing functions. Next, performing the change of variables $r=|x|=R e^{-t / n}$ and $w(t)=$ $n^{\frac{n-1}{n}} \omega_{n-1}^{\frac{1}{n}} u^{*}(r)$, J. Moser reduced (1) to prove the following result: If $p \geq 2$, then there exists a constant $c_{1}$ (depending on $p$ only) such that

$$
\sup _{w \in \mathcal{H}} \int_{0}^{\infty} e^{\rho w^{\frac{p}{p-1}}(t)-t} \mathrm{~d} t \quad\left\{\begin{array}{lll}
\leq c_{1} & \text { if } & \rho \leq 1, \\
=\infty & \text { if } & \rho>1,
\end{array}\right.
$$

where $\mathcal{H}:=\left\{w \in C^{1}([0, \infty)) \quad\right.$ with $\left.\quad w(0)=0, \quad w^{\prime} \geq 0, \quad \int_{0}^{\infty}\left|w^{\prime}\right|^{p} \mathrm{~d} t \leq 1\right\}$.

Motivated by this approach we will analyze a more general class of maximizing problem than (3), which allows us to treat a version of (11) to fractional dimensions. To be precise, the purpose of this paper is three-fold: (I) We investigate TrudingerMoser type inequalities for a class of quasilinear elliptic operators which includes in particular the $p$-Laplacian and $k$-Hessian operators in the radial case; (II) we show that the resulting inequalities are sharp, obtaining the best exponents and (III) we also prove the existence of extremal functions. More explicitly, let $\alpha>0$ and $\beta, \theta \geq 0$ be real numbers. We provide sharp Trudinger-Moser type inequalities for a class of quasilinear elliptic operators in radial form of the type

$$
L u:=-r^{-\theta}\left(r^{\alpha}\left|u^{\prime}(r)\right|^{\beta} u^{\prime}(r)\right)^{\prime}
$$

acting on the weighted Sobolev spaces $X_{R}$ defined as follows: For $0<R \leq \infty$, $q \geq 1$ and $\theta \geq 0$, let us denote by $L_{\theta}^{q}=L_{\theta}^{q}(0, R)$ the weighted Lebesgue space defined as the set of all measurable functions $u$ on $(0, R)$ for which

$$
\|u\|_{L_{\theta}^{q}}:= \begin{cases}\left(\omega_{\theta} \int_{0}^{R} r^{\theta}|u(r)|^{q} \mathrm{~d} r\right)^{\frac{1}{q}}<\infty & \text { if } \quad 1 \leq q<\infty, \\ \operatorname{ess} \sup _{0<r<R}|u(r)|<\infty & \text { if } \quad q=\infty,\end{cases}
$$

where

$$
\omega_{\theta}=2 \pi^{\frac{\theta+1}{2}} / \Gamma\left(\frac{\theta+1}{2}\right), \text { for all } \theta \geq 0,
$$

with $\Gamma(x)=\int_{0}^{\infty} t^{x-1} e^{-t} \mathrm{~d} t$ the Euler gamma function. If $\theta+1=n$ is an integer number, then $\omega_{\theta}$ is the measure of the unit sphere in $\mathbb{R}^{n}$. Denote by $X_{R}$ the space of all locally absolutely continuous functions $u:(0, R] \rightarrow \mathbb{R}$ for which $u(R)=0, u \in$ $L_{\theta}^{\beta+2}$ and $u^{\prime} \in L_{\alpha}^{\beta+2}$. When $R=\infty, u(R)=0$ means $\lim _{r \rightarrow \infty} u(r)=0$. We recall that $X_{R}$ is a Banach space under the norm $\|u\|_{X_{R}}=\left(\|u\|_{L_{\theta}^{\beta+2}}^{\beta+2}+\left\|u^{\prime}\right\|_{L_{\alpha}^{\beta+2}}^{\beta+2}\right)^{\frac{1}{\beta+2}}$.

If $0<R<\infty$, we have

$$
\left(\int_{0}^{R} r^{\theta}|u(r)|^{q} \mathrm{~d} r\right)^{\frac{1}{q}} \leq C\left(\int_{0}^{R} r^{\alpha}\left|u^{\prime}(r)\right|^{\beta+2} \mathrm{~d} r\right)^{\frac{1}{\beta+2}}, u \in X_{R}
$$

provided that one of the following two conditions is fulfilled (cf. [15, page 69]):

(i) For $1 \leq \beta+2 \leq q<\infty$; then, either (a) $\alpha>\beta+1$ and $\theta \geq \alpha \frac{q}{\beta+2}-q \frac{\beta+1}{\beta+2}-1$; or (b) $\alpha \leq \beta+1$ and $\theta \geq 0$,

(ii) For $1 \leq q<\beta+2$; then, either (c) $\alpha>\beta+1$ and $\theta>\alpha \frac{q}{\beta+2}-q \frac{\beta+1}{\beta+2}-1$; or (d) $\alpha \leq \beta+1$ and $\theta \geq 0$. 
By (i) the norm $\|u\|_{X_{R}}$ is equivalent to the norm $\left\|u^{\prime}\right\|_{L_{\alpha}^{\beta+2}}$ for all $\alpha>0$ and $\beta, \theta \geq 0$. Also, combining (a) and (c) in the Sobolev case, that is, under the condition

$$
\alpha-\beta-1>0,
$$

we have the continuous embedding $X_{R} \hookrightarrow L_{\theta}^{q}$ for all $q \in\left(1, q^{*}\right]$, where $q^{*}:=$ $\frac{(\theta+1)(\beta+2)}{\alpha-\beta-1}$ is the Sobolev exponent for this class of spaces (cf. [6]). In this paper, we are interested in the limiting embedding for this class of weighted Sobolev spaces, known as the Trudinger-Moser case:

$$
\alpha-\beta-1=0 \text {. }
$$

In this case, from (b) and (d), we have the continuous embedding $X_{R} \hookrightarrow L_{\theta}^{q}$ for all $q \in[1, \infty)$, but one can see by taking $u(r)=\ln (\ln (e R / r))$ that $X_{R} \nrightarrow L_{\theta}^{\infty}$. Instead, we prove here that $X_{R}$ is embedded in the weighted Orlicz space $L_{\phi}$ determined by $\phi(t)=e^{\mu t^{(\beta+2) /(\beta+1)}}-1$. Let us denote $\mu_{\alpha, \theta}:=(\theta+1) \omega_{\alpha}^{1 / \alpha}$, where $\omega_{\alpha}$ was defined in (5). Our first result reads as follows.

Theorem 1.1. Let $\alpha \geq 1$ and $\beta, \theta \geq 0$ be real numbers and assume that (6) holds. Then there exists $c_{\alpha, \theta}$ depending only on $\alpha, \theta$ and $R$ such that

$$
C_{\alpha, \theta, R}(\mu):=\sup _{u \in X_{R}:\left\|u^{\prime}\right\|_{L_{\alpha}^{\beta+2}} \leq 1} \omega_{\theta} \int_{0}^{R} e^{\mu|u|^{\frac{\beta+2}{\beta+1}}} r^{\theta} \mathrm{d} r \quad\left\{\begin{array}{lll}
\leq c_{\alpha, \theta} & \text { if } \mu \leq \mu_{\alpha, \theta}, \\
=\infty & \text { if } \mu>\mu_{\alpha, \theta} .
\end{array}\right.
$$

In the proof of Theorem 1.1 it is enough to consider $u \in C^{1}, u \geq 0$ and $u(R)=0$. Performing the change of variables $r=R e^{-\frac{t}{\theta+1}}$ and $w(t)=\omega_{\alpha}^{\frac{1}{\alpha+1}}(\theta+1)^{\frac{\alpha}{\alpha+1}} u(r)$, and using (6) it is easy to check that

$$
\omega_{\alpha} \int_{0}^{R} r^{\alpha}\left|u^{\prime}(r)\right|^{\beta+2} \mathrm{~d} r=\int_{0}^{\infty}\left|w^{\prime}(t)\right|^{\beta+2} \mathrm{~d} t
$$

and

$$
\omega_{\theta} \int_{0}^{R} e^{\mu|u|^{\frac{\beta+2}{\beta+1}}} r^{\theta} \mathrm{d} r=\nu_{\theta} \int_{0}^{\infty} e^{\frac{\mu}{\mu_{\alpha, \theta}}|w|^{\frac{\beta+2}{\beta+1}}-t} \mathrm{~d} t
$$

where $\nu_{\theta}=\omega_{\theta} R^{\theta+1} /(\theta+1)$. At this point we emphasize that, since an analogous of the Pólya-Szegö inequality (see (2) ) for $X_{R}$ is not available, we cannot follow the arguments due to J. Moser to reduce (7) to the class of non-increasing functions. However, the complete proof of Theorem 1.1 follows from the next sharpened version of J. Moser's result in (3).

Theorem 1.2. Let $p \geq 2$ be a real number. Then there exists a constant $c_{1}$ depending only on $p$ such that

$$
\sup _{w \in \mathcal{K}} \int_{0}^{\infty} e^{\rho w^{\frac{p}{p-1}}(t)-t} \mathrm{~d} t \quad\left\{\begin{array}{lll}
\leq c_{1} & \text { if } & \rho \leq 1 \\
=\infty & \text { if } & \rho>1
\end{array}\right.
$$

where $\mathcal{K}:=\left\{w \in C^{1}([0, \infty)) \quad\right.$ with $\left.\quad w(0)=0, \quad w \geq 0, \quad \int_{0}^{\infty}\left|w^{\prime}\right|^{p} \mathrm{~d} t \leq 1\right\}$.

We point out that to prove Theorem 1.2 we use a different approach from the one used by J. Moser to analyze this class of maximizing problems. Furthermore, following this approach we establish a new estimate (see Lemma 2.1) allowing us to prove the existence of extremal functions for inequality (7) or, equivalently, (10). 
More precisely,

Theorem 1.3. Under the assumptions of Theorem 1.1, there are extremal functions for $C_{\alpha, \theta, R}(\mu)$ when $\mu \leq \mu_{\alpha, \theta}$; that is, there exists $u \in X_{R}$ such that

$$
C_{\alpha, \theta, R}(\mu)=\omega_{\theta} \int_{0}^{R} e^{\mu|u|^{\frac{\beta+2}{\beta+1}}} r^{\theta} \mathrm{d} r .
$$

The first result on this subject is due to L. Carleson and A. Chang [5], who proved in 1986 that there are extremals for classical Trudinger-Moser inequality (11), when $\Omega$ is a ball in any dimension. In [21 M. Struwe studied the existence of extremal functions for a non-symmetric domain. In the case that $n=2$, he obtained a sufficient condition for the existence of an extremal function, using the blow-up analysis. In 1992, M. Flucher [1] introduced another method, the conformal rearrangement, and derived an isoperimetric inequality, which implies the existence of extremal functions when $\Omega$ is any smooth bounded domain in $\mathbb{R}^{2}$. Latter, K. Lin [17] generalized the existence of an extremal function to any smooth bounded domain in $\mathbb{R}^{n}(n \geq 2)$. See also [18 for related results. Recently, Y. Li [16] obtained the existence of an extremal function for certain Trudinger-Moser inequalities on compact Riemannian manifolds.

Next, we state a Trudinger-Moser type inequality for the unbounded case $X_{\infty}$. For that we introduce the following notation:

$$
p=\beta+2 \quad \text { and } \quad A_{p}(\zeta)=\exp (\zeta)-\sum_{m=0}^{\lfloor p\rfloor} \frac{\zeta^{m}}{m !}
$$

where $\lfloor p\rfloor$ is the largest integer not greater than $p$.

Theorem 1.4. Let $\alpha \geq 1$ and $\beta, \theta \geq 0$ be real numbers and assume that (6) holds. Then for any $\mu \in\left(0, \mu_{\alpha, \theta}\right)$ there is a constant $C$ depending only on $\mu, p$ and $\theta$ such that

$$
\int_{0}^{\infty} A_{p}\left(\mu\left(\frac{|u(r)|}{\left\|u^{\prime}\right\|_{L_{\alpha}^{p}}}\right)^{p /(p-1)}\right) r^{\theta} \mathrm{d} r \leq C\left(\frac{\|u\|_{L_{\theta}^{p}}}{\left\|u^{\prime}\right\|_{L_{\alpha}^{p}}}\right)^{p} \quad \text { for all } \quad u \in X_{\infty} \backslash\{0\} .
$$

We remark that the restriction $\mu<\mu_{\alpha, \theta}$ in (11) is optimal, which excludes $\mu=\mu_{\alpha, \theta}$ in contrast with (7).

Theorem 1.5. For any $\mu \geq \mu_{\alpha, \theta}$ there is a sequence $\left(u_{j}\right) \subset X_{\infty}$ such that $\left\|u_{j}^{\prime}\right\|_{L_{\alpha}^{p}}=$ 1 and

$$
\frac{1}{\left\|u_{j}\right\|_{L_{\theta}^{p}}^{p}} \int_{0}^{\infty} A_{p}\left(\mu\left(\left|u_{j}(r)\right|\right)^{p /(p-1)}\right) r^{\theta} \mathrm{d} r \rightarrow \infty \quad \text { as } \quad j \rightarrow \infty .
$$

Remark 1.6. (1) Extensions of the Trudinger-Moser inequality for unbounded domains have been considered by D. M. Cao 4 in $\mathbb{R}^{2}$ and for any dimension by J. M. do Ó [10. See also S. Adachi and K. Tanaka [1] for a related result. Here we generalize these results for operator (4) on $X_{\infty}$.

(2) Our results improve and complement those in [1,5, 6, 14, 17, 19. Using new arguments and some ideas inspired in [5] and [8], we obtain a new class of Trudinger-Moser inequalities for a more general class of operators.

(3) In view of (7), we can apply variational methods to study the existence and multiplicity of solutions for the following class of quasilinear elliptic 
equations of the form $L u+f(u)=0, u>0$, in $(0, R), u(R)=0$, involving critical growth of Trudinger-Moser type, that is, when $f(s)$ behaves like $e^{\mu s^{(\beta+2) /(\beta+1)}}$ at $\infty$.

(4) For articles involving problems for this class of operator and critical growth of Sobolev type, the reader is referred to the works of P. Clément et al. [6] and D. de Figueiredo et al. 7]. See also the works of J. Jacobsen and K. Schmitt [13, 14, where various results are presented for this class of operator under different conditions on parameters $\alpha, \beta$ and $\theta$.

(5) The following operators, when acting on radially symmetric functions, are simply special cases of the more general class defined by (4):

\begin{tabular}{|l|ccc|}
\hline Operator & $\alpha$ & $\beta$ & $\theta$ \\
\hline Laplacian & $n-1$ & 0 & $n-1$ \\
$p$-Laplacian $(p \geq 2)$ & $n-1$ & $p-2$ & $n-1$ \\
$k$-Hessian & $n-k$ & $k-1$ & $n-1$ \\
\hline
\end{tabular}

In the Trudinger-Moser case (6) we have $n$-Laplacian, $n \geq 2$ and $k$-Hessian with $1 \leq k=n / 2$.

\section{Some PRELIMINARY RESUlts}

For each $\delta>0$ and $p \geq 2$, we define $\mathcal{H}_{\delta}$ by

$$
\mathcal{H}_{\delta}=\left\{\psi:[0, \infty) \rightarrow \mathbb{R} \text { measurable such that } \int_{0}^{\infty}|\psi(s)|^{p} \mathrm{~d} s \leq \delta\right\} .
$$

Also, for all $c>0$ fixed, we denote $L_{c, \delta}$ by

$$
L_{c, \delta}=\sup _{\psi \in \mathcal{H}_{\delta}} \int_{0}^{\infty} \exp \left\{c \int_{0}^{t} \psi(s) \mathrm{d} s-t\right\} \mathrm{d} t .
$$

Let $\Gamma(x)=\int_{0}^{\infty} t^{x-1} e^{-t} \mathrm{~d} t, x>0$, be the gamma Euler function and let $\Psi(x)=$ $\Gamma^{\prime}(x) / \Gamma(x)$ be the psi-function. We point out the following properties of the psifunction which can be found in [3, Theorem 1.2.5]:

$$
\Psi(1)=-\gamma, \quad \Psi(x)-\Psi(1)=\sum_{k=0}^{\infty}\left(\frac{1}{k+1}-\frac{1}{x+k}\right) \quad \text { and } \quad \Psi^{\prime}(x)=\sum_{k=0}^{\infty} \frac{1}{(x+k)^{2}},
$$

where $\gamma:=\lim _{n \rightarrow \infty}\left(\sum_{j=1}^{n} \frac{1}{j}-\log (n)\right)$ is the Euler constant. In this way we obtain

Lemma 2.1. For each $c>0$, the following properties hold for the supremum $L_{c, \delta}$ :

a) $L_{c, \delta}$ is finite.

b) $L_{c, \delta}$ is attained by a non-negative function $\psi_{0} \in \mathcal{H}_{\delta}$ with $\psi_{0} \not \equiv 0$.

c) $L_{c, \delta} \leq \exp \left\{\left(\frac{p-1}{p}\right)^{p-1} \frac{c^{p} \delta}{p}+\Psi(p)+\gamma\right\}$.

Proof. a) By the definition of $L_{c, \delta}$, it is obvious that we may assume $\psi \geq 0$ since we can replace $\psi$ by $|\psi|$ without decreasing the integral. For all $\psi \in \mathcal{H}_{\delta}$ and $A \geq 0$, for $t \geq A$ we have

$$
\int_{0}^{t} \psi(s) \mathrm{d} s \leq \delta^{1 / p} A^{(p-1) / p}+\delta^{1 / p}(t-A)^{(p-1) / p} .
$$


Since $\int_{0}^{t} \psi(s) \mathrm{d} s \leq \delta^{1 / p} t^{(p-1) / p}$ and $\lim _{x \rightarrow \infty} x^{(p-1) / p} / x=0$, choosing $N \geq 2^{p} c^{p} \delta+A$ we have that

$$
c \int_{0}^{t} \psi(s) \mathrm{d} s \leq c \delta^{1 / p} A^{(p-1) / p}+(t-A) / 2, \quad \text { for all } \quad t \geq N .
$$

Hence, for $\epsilon>0$, choosing $N \geq \max \left\{2^{p} c^{p} \delta+A,-2 \log (\epsilon / 2)-A+2 c \delta^{1 / p} A^{(p-1) / p}\right\}$ we have

$$
\int_{N}^{\infty} e^{c \int_{0}^{t} \psi(s) \mathrm{d} s-t} \mathrm{~d} t \leq 2 e^{c \delta^{1 / p} A^{(p-1) / p}-A / 2} e^{-N / 2} \leq \epsilon \quad \text { for any } \quad \psi \in \mathcal{H}_{\delta} .
$$

On the other hand, for any $\psi \in \mathcal{H}_{\delta}$ it holds that $\int_{0}^{t} \psi(s) \mathrm{d} s \leq \delta^{1 / p} N^{(p-1) / p}$ if $t \leq N$. Therefore $L_{c, \delta}$ is finite.

b) For $\psi \in \mathcal{H}_{\delta}$, denote

$$
I(\psi)=\int_{0}^{\infty} \exp \left\{c \int_{0}^{t} \psi(s) \mathrm{d} s-t\right\} \mathrm{d} t \text { and } I_{N}(\psi)=\int_{0}^{N} \exp \left\{c \int_{0}^{t} \psi(s) \mathrm{d} s-t\right\} \mathrm{d} t .
$$

Let $\left(\psi_{j}\right) \subset \mathcal{H}_{\delta}$ be a sequence such that $I\left(\psi_{j}\right)$ converges to $L_{c, \delta}$. In particular, $\left(\psi_{j}\right)$ is bounded in $L^{p}[0, \infty)$; thus up to a subsequence, we have $\psi_{j} \rightarrow \psi_{0}$ weakly in $L^{p}[0, \infty)$, and hence, by the weak lower semicontinuity of norm, we have $\psi_{0} \in \mathcal{H}_{\delta}$. Consequently, for $N>0$ we have $\left.\left.\psi_{j}\right|_{[0, N]} \rightarrow \psi_{0}\right|_{[0, N]}$ weakly in $L^{p}[0, N]$, and this implies $\lim _{j \rightarrow \infty} \int_{0}^{t} \psi_{j}(s) \mathrm{d} s=\int_{0}^{t} \psi_{0}(s) \mathrm{d} s$ for all $t$ in $[0, N]$ (cf. [20, Theorem 11]). Given $\epsilon>0$, choose $N$ satisfying (13). Since $\int_{0}^{t} \psi_{j}(s) \mathrm{d} s \leq \delta^{1 / p} N^{(p-1) / p}$ on $[0, N]$, using Lebesgue's Convergence Theorem, we obtain $\lim _{j \rightarrow \infty} I_{N}\left(\psi_{j}\right)=I_{N}\left(\psi_{0}\right) \geq$ $I_{N}\left(\psi_{j}\right)-\epsilon$, for $j$ sufficiently large, which together with (13) implies $I\left(\psi_{0}\right) \geq$ $I_{N}\left(\psi_{0}\right) \geq I_{N}\left(\psi_{j}\right)-\epsilon \geq I\left(\psi_{j}\right)-2 \epsilon$. Letting $j \rightarrow \infty$, we get $I\left(\psi_{0}\right) \geq L_{c, \delta}-2 \epsilon$ for all $\epsilon>0$, which implies that $I\left(\psi_{0}\right)=L_{c, \delta}$. Taking $a>0$ and $\psi_{a}:[0, \infty) \rightarrow \mathbb{R}$ given by $\psi_{a}(t)=a^{1 / p}$ if $0 \leq t \leq t_{a}, \psi_{a}(t)=\left(a-d_{a}\left(t-t_{a}\right)\right)^{1 / p}$ if $t_{a} \leq t \leq t_{a}+a / d_{a}$ and $\psi_{a}(t)=0$ if $t \geq t_{a}+a / d_{a}$, one can choose $a, t_{a}$ and $d_{a}$ such that $\psi_{a} \in \mathcal{H}_{\delta}$ and $I\left(\psi_{a}\right)>1$. Therefore our extremal function $\psi_{0}$ is non-trivial since $I(0)=1$.

c) The proof of this fact is analogous to [5, Lemma 1]. Here, we include the outline of the proof for completeness. First, note that

$$
L_{c, \delta}=\sup _{\psi \in \mathcal{S}_{\delta}} \int_{0}^{\infty} e^{c \int_{0}^{t} \psi(s) \mathrm{d} s-t} \mathrm{~d} t
$$

where $\mathcal{S}_{\delta}=\left\{\psi \in L^{p}[0, \infty) ; \int_{0}^{\infty}|\psi|^{p} \mathrm{~d} t=\delta\right\}$. Let $\psi_{0} \in \mathcal{H}_{\delta}$ be an extremal function ensured in b). We can suppose $\psi_{0} \geq 0$ and $\psi_{0} \not \equiv 0$. By the Lagrange multipliers theorem there exists a constant $\bar{\lambda}>0$ such that, for all $\varphi \in L^{p}(0, \infty)$,

$$
\int_{0}^{\infty} e^{c \int_{0}^{t} \psi_{0}(s) \mathrm{d} s-t}\left(\int_{0}^{t} \varphi(s) \mathrm{d} s\right) \mathrm{d} t=\bar{\lambda} \int_{0}^{\infty}\left|\psi_{0}(t)\right|^{p-2} \psi_{0}(t) \varphi(t) \mathrm{d} t .
$$

In particular, for all $h \in C_{0}^{1}(0, \infty)$,

$$
\int_{0}^{\infty} e^{c \int_{0}^{t} \psi_{0}(s) \mathrm{d} s-t} h(t) \mathrm{d} t=\bar{\lambda} \int_{0}^{\infty}\left|\psi_{0}(t)\right|^{p-2} \psi_{0}(t) h^{\prime}(t) \mathrm{d} t .
$$

Since $e^{c \int_{0}^{t} \psi_{0}(s) \mathrm{d} s-t} \in C[0, \infty)$, its indefinite integral $F(t)=\int_{0}^{t} e^{c \int_{0}^{s} \psi_{0}(\tau) \mathrm{d} \tau-s} \mathrm{~d} s$ is a $C^{1}$ function in $[0, \infty)$ and $F^{\prime}(t)=e^{c \int_{0}^{t} \psi_{0}(s) \mathrm{d} s-t}$. By (14), since $h \in C_{0}^{1}(0, \infty)$,

$$
\int_{0}^{\infty}\left(F+\bar{\lambda}\left|\psi_{0}\right|^{p-2} \psi_{0}\right) h^{\prime} \mathrm{d} t=\int_{0}^{\infty}\left(F h^{\prime}+F^{\prime} h\right) \mathrm{d} t=\int_{0}^{\infty} \frac{\mathrm{d}}{\mathrm{d} t}(F(t) h(t)) \mathrm{d} t=0 .
$$


Thus, from the Dubois-Reymond Lemma we have $F+\bar{\lambda}\left|\psi_{0}\right|^{p-2} \psi_{0}=C$ a.e. in $[0, \infty)$ and it follows that $\varphi=C-F$ is a $C^{1}$ function and satisfies $\varphi=\bar{\lambda}\left|\psi_{0}\right|^{p-2} \psi_{0}$ a.e. in $[0, \infty)$. Also, $\varphi^{\prime}=-F^{\prime}<0$ on $[0, \infty)$. Since $\psi_{0} \geq 0$ we have also that $\varphi \geq 0$ a.e. in $[0, \infty)$. If $\varphi\left(t_{0}\right)=0$ for some $t_{0}>0$, then $\varphi(t)=\int_{t_{0}}^{t} \varphi^{\prime}(s) \mathrm{d} s<0$ for all $t>t_{0}$ which contradicts $\varphi \geq 0$ a.e. in $[0, \infty)$. Therefore, $\varphi>0$ and $\psi(t)=(\varphi / \bar{\lambda})^{\frac{1}{p-1}}(t)$ is a $C^{1}$ function with $\psi=\psi_{0}$ a.e. in $[0, \infty)$. Thus, we have $I(\psi)=I\left(\psi_{0}\right)$. Hence, we can take a $C^{1}$ extremal function $\psi$ for $L_{c, \delta}$. Moreover, since $\varphi^{\prime}=-F^{\prime}$ we obtain

$$
e^{c \int_{0}^{t} \psi(s) \mathrm{d} s-t}=\lambda_{0} \psi^{\prime}(t)(\psi(t))^{p-2}
$$

for some constant $\lambda_{0}$. Setting $v(t)=c \int_{0}^{t} \psi(s) \mathrm{d} s-t$, the above equation becomes

$$
e^{v(t)}=\lambda v^{\prime \prime}(t)\left(1+v^{\prime}(t)\right)^{p-2},
$$

where $\lambda=\lambda_{0} c^{1-p}$. Moreover, $v$ satisfies $\int_{0}^{\infty}\left(1+v^{\prime}(t)\right)^{p} \mathrm{~d} t=c^{p} \delta$ and

$$
v(0)=0, \quad v^{\prime}(\infty)=-1 \quad \text { and } \quad v(\infty)=-\infty .
$$

From (15), using integration by parts, we have

$$
e^{v(t)}=\frac{\lambda}{p}\left(1+v^{\prime}(t)\right)^{p}-\frac{\lambda}{p-1}\left(1+v^{\prime}(t)\right)^{p-1}+C .
$$

Using (16) and letting $t \rightarrow \infty$, we get $C=0$. Hence combining the above equation with (15), we obtain

$$
v^{\prime \prime}(t)=\frac{1}{p}\left(1+v^{\prime}(t)\right)^{2}-\frac{1}{p-1}\left(1+v^{\prime}(t)\right) .
$$

Integrating this last equation gives

$$
1+v^{\prime}(t)=\frac{p}{p-1}\left(1+B e^{t /(p-1)}\right)^{-1},
$$

where $B \geq 0$ is chosen such that

$$
c^{p} \delta=\int_{0}^{\infty}\left(1+v^{\prime}(t)\right)^{p} \mathrm{~d} t .
$$

Taking into account (18) and (19), by performing a change-of-variables $u=1+$ $B e^{t /(p-1)}$, we obtain

$$
\begin{aligned}
& c^{p} \delta=\left(\frac{p}{p-1}\right)^{p}(p-1) \int_{B+1}^{\infty} \frac{\mathrm{d} u}{(u-1) u^{p}}=\left(\frac{p}{p-1}\right)^{p}(p-1) \sum_{k=0} \frac{1}{(k+p)(B+1)^{k+p}} \\
& (20) \geq\left(\frac{p}{p-1}\right)^{p}(p-1)[\log (1+1 / B)-(\Psi(p)+\gamma)],
\end{aligned}
$$

where in this last estimate we use the properties of the psi-function pointed out in (12). On the other hand, taking $t=0$ in (17) and using (16) and (18), we can write

$$
1=\frac{\lambda}{p}\left(\frac{p}{p-1} \cdot \frac{1}{1+B}\right)^{p}-\frac{\lambda}{p-1}\left(\frac{p}{p-1} \cdot \frac{1}{1+B}\right)^{p-1} .
$$

From (16) and (18), integrating (15) yields

$$
\int_{0}^{\infty} e^{v(t)} \mathrm{d} t=\frac{\lambda}{p-1} \int_{0}^{\infty} \frac{\mathrm{d}}{\mathrm{d} t}\left(1+v^{\prime}(t)\right)^{p-1} \mathrm{~d} t=-\frac{\lambda}{p-1}\left(\frac{p}{p-1} \cdot \frac{1}{1+B}\right)^{p-1}
$$

which together with (20) and (21) gives the desired estimate. 
Remark 2.2. We point out that Lemma 2.1 improves Lemma 1 in [5], and therefore all of its consequences. For example, the next result is a sharpened version of Lemma 2 in [5]: Here the monotonicity hypothesis under $w$ is not required and we give a new estimate in which the integer number $n$ can be replaced by an arbitrary real number $p \geq 2$. More explicitly,

Lemma 2.3. Let $p \geq 2, a>0$ and $\delta>0$ be real numbers. For each $w$ non-negative $C^{1}$ function on $[0, \infty)$ satisfying $\int_{a}^{\infty}\left|w^{\prime}(t)\right|^{p} \mathrm{~d} t \leq \delta$ we have

$$
\int_{a}^{\infty} e^{w^{q}(t)-t} \mathrm{~d} t \leq e^{w^{q}(a)-a} \frac{1}{1-\delta^{1 /(p-1)}} \exp \left\{\left(\frac{p-1}{p}\right)^{p-1} \frac{c^{p} \gamma_{p}}{p}+\Psi(p)+\gamma\right\},
$$

where $\gamma_{p}=\delta\left(1-\delta^{1 /(p-1)}\right)^{1-p}, c=q w^{q-1}(a)$ and $q=p /(p-1)$.

Proof. Let $w$ be a function under the above hypotheses. Set $x=t-a$ and $\psi(x)=$ $w(x+a)-w(a)$; then $w(t)=\psi(x)+w(a) \geq 0$ for all $x \geq 0$. Note that if $1<q \leq 2$ and $d \geq 0$, we have $(s+d)^{q} \leq d^{q}+q d^{q-1} s+|s|^{q}$ for all $s \geq-d$. Thus,

$$
w(t)^{q}=(\psi(x)+w(a))^{q} \leq w(a)^{q}+q w(a)^{q-1} \psi(x)+|\psi(x)|^{q} .
$$

Since $\psi(0)=0$ and $\int_{0}^{\infty}\left|\psi^{\prime}(x)\right|^{p} \mathrm{~d} x \leq \delta$, by Hölder it follows that $|\psi(x)|^{q} \leq \delta^{1 /(p-1)} x$. Therefore, using estimate (22) we obtain

$$
\int_{a}^{\infty} e^{w^{q}(t)-t} \mathrm{~d} t \leq e^{w^{q}(a)-a} \frac{1}{1-\delta^{1 /(p-1)}} \int_{0}^{\infty} e^{c \varphi(y)-y} \mathrm{~d} y,
$$

where $y=\left(1-\delta^{1 /(p-1)}\right) x, c=q w^{q-1}(a)$ and $\varphi(y)=\psi(x)$. Since

$$
\int_{0}^{\infty}\left|\varphi^{\prime}(y)\right|^{p} \mathrm{~d} y=\left(1-\delta^{1 /(p-1)}\right)^{1-p} \int_{0}^{\infty}\left|\psi^{\prime}(x)\right|^{p} \mathrm{~d} x \leq \gamma_{p},
$$

the result follows by Lemma 2.1

\section{Proofs of Theorems 1.1 and 1.2}

As we already mentioned, in view of reduction (8)-(9), Theorem 1.1 is an immediate consequence of Theorem 1.2. Let $p \geq 2$ and $q=p /(p-1)$ be the Hölder conjugate of $p$. First, we prove that for any $\rho>0$ and $w \in \mathcal{K}$ we have

$$
\int_{0}^{\infty} e^{\rho w^{q}(t)-t} \mathrm{~d} t<\infty
$$

Notice that given $\epsilon>0$, there exists $T=T(\epsilon)$ such that $\int_{T}^{\infty}\left|w^{\prime}(t)\right|^{p} \mathrm{~d} t<\epsilon$. Thus, the Hölder inequality implies

$$
w(t) \leq w(T)+\epsilon^{1 / p}(t-T)^{1 / q}, \quad \text { for all } t \geq T .
$$

Hence, $\lim _{t \rightarrow \infty} w(t) / t^{1 / q}=0$. Thus, we have $\rho w^{q}(t)<t / 2$, for $t$ sufficiently large, which implies that (23) holds. Next, we show that (23) is uniformly bounded for $w \in \mathcal{K}$ under the condition $\rho \leq 1$. If $\rho<1$ it is easier. Indeed, in this case, for any $w \in \mathcal{K}$ we have

thus

$$
w(t)=\int_{0}^{t} w^{\prime}(s) \mathrm{d} s \leq\left(\int_{0}^{t}\left|w^{\prime}(s)\right|^{p} \mathrm{~d} s\right)^{1 / p} t^{1 / q} \leq t^{1 / q}
$$

$$
\int_{0}^{\infty} e^{\rho w^{q}(t)-t} \mathrm{~d} t \leq \int_{0}^{\infty} e^{(\rho-1) t} \mathrm{~d} t \leq 1 /(1-\rho) .
$$


Now, suppose that $\rho=1$ and $w \in \mathcal{K}$. We have two cases to analyze:

Case $(\mathrm{a}): w^{q}(t) \leq t-2 \ln ^{+} t$ for all $t \in[1, \infty)$. In this case we have

$$
\int_{0}^{\infty} e^{w^{q}(t)-t} \mathrm{~d} t \leq \int_{0}^{\infty} e^{-2 \ln ^{+} t} \mathrm{~d} t \leq 2
$$

Case (b): There exists $a \in[1, \infty)$, the smallest number, such that $w^{q}(a)=a-2 \ln ^{+} a$. We set $\delta=\int_{a}^{\infty}\left|w^{\prime}(t)\right|^{p} \mathrm{~d} t$. Using Lemma 2.3, we obtain

$$
\int_{a}^{\infty} e^{w^{q}(t)-t} \mathrm{~d} t \leq \frac{1}{1-\delta^{1 /(p-1)}} e^{K+\Psi(p)+\gamma}
$$

where $K=w^{q}(a)\left(1+\frac{1}{p-1} \frac{\delta}{\left(1-\delta^{1 /(p-1)}\right)^{p-1}}\right)-a$. Also,

$$
w^{q}(a) \leq\left(\int_{0}^{a}\left|w^{\prime}(s)\right|^{p} \mathrm{~d} s\right)^{q / p} a \leq(1-\delta)^{1 /(p-1)} a,
$$

which implies

$$
\delta \leq 1-\left(1-\frac{2 \ln ^{+} a}{a}\right)^{p-1} \leq(p-1) \frac{2 \ln ^{+} a}{a}
$$

because $w^{q}(a)=a-2 \ln ^{+} a$ and $1-t^{d} \leq d(1-t), \quad t \geq 0$ for any $d \geq 1$. Note that $K=a\left(1-\delta^{1 /(p-1)}\right)^{1-p} F(a)$, where

$$
F(a)=\frac{\delta}{p-1}-\frac{\delta}{p-1} \frac{2 \ln ^{+} a}{a}-\frac{2 \ln ^{+} a}{a}\left(1-\delta^{\frac{1}{p-1}}\right)^{p-1} .
$$

Since $1-\left(1-\delta^{\frac{1}{p-1}}\right)^{p-1} \leq(p-1) \delta^{\frac{1}{p-1}}$, (25) yields

$$
F(a) \leq \frac{2 \ln ^{+} a}{a}\left[1-\left(1-\delta^{\frac{1}{p-1}}\right)^{p-1}-\frac{\delta}{p-1}\right] \leq(p-1)^{\frac{p}{p-1}}\left(\frac{2 \ln ^{+} a}{a}\right)^{\frac{p}{p-1}} .
$$

Thus, it follows that

$$
K \leq \frac{(p-1)^{q}}{\left(1-\delta^{1 /(p-1)}\right)^{p-1}} \cdot a\left(\frac{2 \ln ^{+} a}{a}\right)^{q} .
$$

Since $e \ln a \leq a$ for all $a>0$, using (25) we get $\delta \leq 1-\left(1-\frac{2}{e}\right)^{p-1}:=d_{p}$; then

$$
\frac{1}{1-\delta^{1 /(p-1)}} \leq \frac{1}{1-d_{p}{ }^{1 /(p-1)}} \text {. }
$$

Since $g(x)=x\left(\frac{\ln x}{x}\right)^{q}$ is uniformly bounded on $[1, \infty)$ by some constant depending only on $p$, by estimates (24), (26) and (27) we conclude that $\int_{a}^{\infty} e^{w^{q}(t)-t} \mathrm{~d} t \leq c_{1}$. This estimate and the inequality $\int_{0}^{a} e^{w^{q}(t)-t} \mathrm{~d} t \leq 2$ shows that (23) is uniformly bounded for $w \in \mathcal{K}$ when $\rho \leq 1$.

Finally we verify that $\mu_{\alpha, \theta}$ in Theorem 1.1 is optimal or, equivalently, $\rho=1$ is optimal in Theorem 1.2. For this, we consider Moser's functions:

$$
w_{j}(t)=\left\{\begin{array}{lll}
\frac{t}{j^{1 / p}} & \text { if } \quad 0 \leq t \leq j, \\
j^{1 / q} & \text { if } \quad t \geq j .
\end{array}\right.
$$

It is clear that $\int_{0}^{\infty}\left|w_{j}^{\prime}(t)\right|^{p} \mathrm{~d} t=1$ and $\int_{0}^{\infty} e^{\rho w_{j}^{q}(t)-t} \mathrm{~d} t \geq e^{(\rho-1) j} \rightarrow \infty$ if $\rho>1$. 


\section{Proof of Theorem 1.3}

As we already mentioned, because of the reduction (8)-(9), it is enough to show that there exists an extremal for (10) when $\rho \leq 1$. For $\rho<1$, we can use similar arguments to those in (i) below. Indeed, assertion (28) below is almost immediate and it implies the existence of extremals. Assume $\rho=1$ and let $q=p /(p-1)$ be the Hölder conjugate of $p \geq 2$. We set

$$
J(w)=\int_{0}^{\infty} e^{w^{q}(t)-t} \mathrm{~d} t, \forall w \in \mathcal{K} \quad \text { and } \quad \mathcal{I}_{p}:=\sup _{w \in \mathcal{K}} J(w),
$$

where $\mathcal{K}$ was defined in Theorem 1.2. In order to prove the existence of extremal (10) we will show that $\mathcal{I}_{p}$ is attained by some $w_{0} \in \mathcal{K}$. For this, let $\gamma$ and $\Psi(p)$ be as in (12). Here, we use the two-step strategy of Carleson and Chang (cf. [5]):

- Step 1: Assuming has no extremal for the supreme in $\mathcal{I}_{p}$, then we prove that $\mathcal{I}_{p} \leq 1+e^{\Psi(p)+\gamma}$.

- Step 2: For each $p \geq 2$, we find a specific function $f_{p} \in \mathcal{K}$ such that $J\left(f_{p}\right)>1+e^{\Psi(p)+\gamma}$, which is a contraction.

To verify Step 1, we use Lemma 2.3 Let $\left(w_{j}\right) \subset \mathcal{K}$ be a sequence such that $J\left(w_{j}\right)$ converges to $\mathcal{I}_{p}$. Assuming that there is no extremal for $\mathcal{I}_{p}$, we claim that, up to subsequence, $\left(w_{j}\right)$ satisfies:

(i) For each $A>0, \int_{0}^{A}\left|w_{j}^{\prime}(t)\right|^{p} \mathrm{~d} t \rightarrow 0$ as $j \rightarrow \infty$.

(ii) There exists $a_{j}$ in $[1, \infty)$ such that $w_{j}^{q}\left(a_{j}\right)=a_{j}-2 \ln a_{j}$ and $\lim _{j \rightarrow \infty} a_{j}=\infty$.

(iii) $\limsup \int_{j}^{\infty} e^{w_{j}^{q}(t)-t} \mathrm{~d} t \leq e^{\Psi(p)+\gamma}$.

(iv) $\lim _{j} \int_{0}^{a_{j}} e^{w_{j}^{q}(t)-t} \mathrm{~d} t=1$.

It is clear that the Step 1 follows by (iii) and (iv).

(i). We argue by contradiction. Suppose that (i) is false. In other words, there exist constants $A>0$ and $0<\delta<1$ such that

$$
\int_{0}^{A}\left|w_{j}^{\prime}(t)\right|^{p} \mathrm{~d} t \geq \delta \quad \text { for } \quad j \geq j_{0} .
$$

Then $\int_{A}^{\infty}\left|w_{j}^{\prime}(t)\right|^{p} \mathrm{~d} t \leq 1-\delta$, which implies $w_{j}(t)-w_{j}(A) \leq(1-\delta)^{1 / p}(t-A)^{1 / q}$, $t \geq A$. By elementary calculus, for each $a \geq 0,1<p \leq 2$ and $0<\kappa<1$, we have

$$
(a+x)^{p} \leq a^{p}+p a^{p-1} x+x^{p}, \quad x \geq 0 \quad \text { and } \quad \lim _{x \rightarrow \infty} \frac{x^{\kappa}}{x}=0 .
$$

Thus, for $\epsilon>0$ we can choose $N>A$ such that $w_{j}(t)^{q} \leq(1-\delta / 2)^{1 /(p-1)}(t-A)+q A$, $t>N$, which implies

$$
\int_{N}^{\infty} e^{w_{j}^{q}(t)-t} \mathrm{~d} t \leq e^{q A} \frac{1}{1-(1-\delta / 2)^{1 /(p-1)}} e^{\left((1-\delta / 2)^{1 /(p-1)}-1\right) N} \leq \epsilon
$$

for $N$ is sufficiently large and $j \geq j_{0}$. As such, as in item b) of Lemma 2.1 and using [20. Theorem 11], up to a subsequence, we can assume that $w_{j}$ converges pointwise to $w_{0} \in \mathcal{K}$. For $0 \leq t \leq N$ one has $w_{j}(t) \leq N^{1 / q}$. Then by Lebesgue's Convergence Theorem

$$
\mathcal{J}_{N}\left(w_{0}\right):=\int_{0}^{N} e^{w_{0}{ }^{q}(t)-t} \mathrm{~d} t=\lim _{j} \int_{0}^{N} e^{w_{j}^{q}(t)-t} \mathrm{~d} t=\lim _{j} \mathcal{J}_{N}\left(w_{j}\right) \geq \mathcal{J}_{N}\left(w_{j}\right)-\epsilon
$$


when $j$ is large. By (28) it follows that $J\left(w_{0}\right) \geq \mathcal{J}_{N}\left(w_{0}\right) \geq \mathcal{J}_{N}\left(w_{j}\right)-\epsilon \geq J\left(w_{j}\right)-2 \epsilon$. For $j \rightarrow \infty$ we get $J\left(w_{0}\right) \geq \mathcal{I}_{p}-2 \epsilon$ for all $\epsilon>0$. Then we obtain $J\left(w_{0}\right)=\mathcal{I}_{p}$. But this leads to a contradiction with the assumption of Step 1, and so (i) holds.

(ii). We first claim that, for $j$ sufficiently large, there exists $a_{j} \in[1, \infty)$ satisfying $w_{j}^{q}\left(a_{j}\right)=a_{j}-2 \ln a_{j}$. Since $w_{j}^{q}(t) \leq t-2 \ln ^{+} t=t$ for all $t \in[0,1]$, if we assume that our claim is false, we have that $w_{j}^{q}(t)<t-2 \ln ^{+} t$ for all $t \in[1, \infty)$. Thus,

$$
J\left(w_{j}\right)=\int_{0}^{\infty} e^{w_{j}^{q}(t)-t} \mathrm{~d} t \leq 1+\int_{1}^{\infty} e^{-2 \ln t} \mathrm{~d} t=2
$$

which implies that $\mathcal{I}_{p} \leq \lim \sup _{j} J\left(w_{j}\right) \leq 2$. This contradicts Step 2 .

Now, by (i), given $A>0$, we can choose $j_{0}=j_{0}(A)$ such that

$$
\left(\int_{0}^{A}\left|w_{j}^{\prime}(s)\right|^{p} \mathrm{~d} s\right)^{1 /(p-1)}<\frac{e-2}{e} \text { for all } j \geq j_{0} .
$$

Thus, by Hölder's inequality and since $\frac{e-2}{e} t \leq t-2 \ln ^{+} t$ for all $t \in[0, \infty)$,

$$
w_{j}^{q}(t) \leq\left(\int_{0}^{A}\left|w_{j}^{\prime}(s)\right|^{p} \mathrm{~d} s\right)^{1 /(p-1)} t<\frac{e-2}{e} t \leq t-2 \ln ^{+} t,
$$

for all $0 \leq t \leq A$ and $j \geq j_{0}$, which implies that $a_{j} \geq A$ for all $j \geq j_{0}$. Therefore, $\lim _{j \rightarrow \infty} a_{j}=\infty$.

(iii). This follows from Lemma 2.3. Indeed, choosing $w=w_{j}, a=a_{j}, \delta=\delta_{j}=$ $\int_{a_{j}}^{\infty}\left|w_{j}^{\prime}(t)\right|^{p} \mathrm{~d} t$ and applying Lemma 2.3, we obtain

$$
\int_{a_{j}}^{\infty} e^{w_{j}^{q}(t)-t} \mathrm{~d} t \leq \frac{1}{1-\delta_{j}^{1 /(p-1)}} e^{K_{j}+\Psi(p)+\gamma},
$$

where

$$
K_{j}=w_{j}^{q}\left(a_{j}\right)\left[1+\frac{\delta_{j}}{(p-1)\left(1-\delta_{j}^{1 /(p-1)}\right)^{p-1}}\right]-a_{j} .
$$

Thus, the proof of (iii) is completed by showing that $\delta_{j} \rightarrow 0$ and $K_{j} \rightarrow 0$ as $j \rightarrow \infty$. Since $w_{j} \in \mathcal{K}$ we have $w_{j}^{q}\left(a_{j}\right) \leq\left(1-\delta_{j}\right)^{1 /(p-1)} a_{j}$, which implies $\delta_{j} \rightarrow 0$, because

$$
\delta_{j} \leq 1-\left(1-\frac{2 \ln ^{+} a_{j}}{a_{j}}\right)^{p-1} \leq(p-1) \frac{2 \ln ^{+} a_{j}}{a_{j}} \rightarrow 0 \quad \text { as } \quad j \rightarrow \infty,
$$

since $a_{j} \rightarrow \infty$ by (ii). We also have, for all $j \geq j_{0}$,

$$
K_{j} \leq w_{j}^{q}\left(a_{j}\right)\left[1+\frac{\delta_{j}}{p-1}+\frac{2 p \delta_{j}^{p /(p-1)}}{p-1}\right]-a_{j} \leq 2(p-1)^{q} a_{j}\left(\frac{2 \ln ^{+} a_{j}}{a_{j}}\right)^{q}
$$

which implies $K_{j} \rightarrow 0$ as $j \rightarrow \infty$.

(iv). It follows from (i) that $w_{j} \rightarrow 0$ uniformly on compact sets. Thus, given $\epsilon>0$ and $A>0$, we have $w_{j}(t)^{q} \leq \epsilon$ for all $0 \leq t \leq A$ and $j$ is sufficiently large. 
Since $a_{j}$ is the smallest number such that $w_{j}(t)^{q} \geq t-2 \ln ^{+} t$, we obtain

$$
\int_{0}^{a_{j}} e^{w_{j}(t)^{q}-t} \mathrm{~d} t=\int_{0}^{A} e^{w_{j}(t)^{q}-t} \mathrm{~d} t+\int_{A}^{a_{j}} e^{w_{j}(t)^{q}-t} \mathrm{~d} t \leq e^{\epsilon}\left(1-\frac{1}{e^{A}}\right)+\left(\frac{1}{A}-\frac{1}{a_{j}}\right) \leq 1,
$$

for $\epsilon$ sufficiently small and $A$ sufficiently large. On the other hand,

$$
\int_{0}^{a_{j}} e^{w_{j}(t)^{q}-t} \mathrm{~d} t \geq \int_{0}^{a_{j}} e^{-t} \mathrm{~d} t=1-e^{-a_{j}} \rightarrow 1 \quad \text { as } \quad j \rightarrow \infty,
$$

which completes the proof of (iv) and Step 1 of the theorem.

The proof of the Step 2 consists in the construction of a function $f_{p} \in \mathcal{K}$ such that

$$
f_{p}(t)=\left\{\begin{array}{lll}
\frac{1}{q}(p-1)^{-1 / p} t & \text { if } \quad 0 \leq t \leq p \\
(t-1)^{1 / q} & \text { if } \quad p \leq t \leq N_{p} \\
\left(N_{p}-1\right)^{1 / q} & \text { if } \quad t \geq N_{p}
\end{array}\right.
$$

where $N_{p}$ was chosen such that $\int_{0}^{\infty} f_{p}^{\prime}(t)^{p} \mathrm{~d} t=1$. Explicitly, $N_{p}=(p-1) e^{q^{p}-q}+1$. It is easy to see that

$$
J\left(f_{p}\right)=\int_{0}^{p} \exp \left\{q^{-q}(p-1)^{-1 /(p-1)} t^{q}-t\right\} \mathrm{d} t+(p-1) e^{q^{p}-q-1}+\frac{2-p}{e} .
$$

Considering the change of variable $v=t / p$, we have

$$
J\left(f_{p}\right)=p \int_{0}^{1} e^{h(v)} \mathrm{d} v+(p-1) e^{q^{p}-q-1}+\frac{2-p}{e},
$$

where $h(v)=(p-1) v^{q}-p v$. Clearly, $h(v)>-p v$ on $(0,1 / p]$ and $h(v) \geq-1$ on $[1 / p, 1]$. Thus,

$$
p \int_{0}^{1} e^{h(v)} \mathrm{d} v>p \int_{0}^{1 / p} e^{-p v} \mathrm{~d} v+\frac{e}{p} \int_{1 / p}^{1} \mathrm{~d} v=1-\frac{2-p}{e} .
$$

Hence $J\left(f_{p}\right)>1+(p-1) e^{q^{p}-q-1}$. The proof is completed by showing that $(p-$ 1) $e^{q^{p}-q-1} \geq e^{\Psi(p)+\gamma}, p \geq 2$. To see this fact consider the auxiliary function $\xi$ : $[2, \infty) \rightarrow \mathbb{R}$ defined by $\xi(p)=\Psi(p)+\gamma-\log (p-1)-q^{p}+q+1$. Note that $\xi(2)=0$ and $\xi^{\prime}(p) \leq 0$, for all $p \in(2, \infty)$. Indeed, from (12),

$$
\Psi^{\prime}(p)=\sum_{k=0}^{\infty} \frac{1}{(k+p)^{2}} \leq \sum_{k=0}^{\infty} \frac{1}{\left(k+p-\frac{1}{2}\right)\left(k+p+\frac{1}{2}\right)}=\frac{1}{\left(p-\frac{1}{2}\right)}
$$

and $\frac{d}{d p}\left(q^{p}-q\right)=q^{p}(\log (q)-q+1)+(q-1)^{2}$. Finally, after subtle estimates of the terms $q^{p}$ and $\log q$ we get $1 /(p-1 / 2) \leq q^{p}(\log (q)-q+1)+(q-1)^{2}+(q-1)$, which completes the proof of Theorem 1.3

\section{Proof of Theorem 1.4}

Let $u \in X_{\infty}$ be a $C^{1}$ function, and set $r=e^{-\frac{t}{\theta+1}}$ and $w(t)=\omega_{p-1}^{\frac{1}{p}}(\theta+1)^{\frac{1}{q}} u(r)$ with $q=p /(p-1)$. Then $w(t)$ is defined on the interval $(-\infty, \infty)$ and satisfies

$$
\lim _{t \rightarrow-\infty} w(t)=0
$$


Taking into account that $p=\beta+2$ and (6), we obtain

$$
\begin{aligned}
\omega_{\alpha} \int_{0}^{\infty} r^{\alpha}\left|u^{\prime}(r)\right|^{p} \mathrm{~d} r & =\int_{-\infty}^{\infty}\left|w^{\prime}(t)\right|^{p} \mathrm{~d} t \\
\omega_{\theta} \int_{0}^{\infty} r^{\theta}|u(r)|^{p} \mathrm{~d} r & =\frac{\omega_{\theta}}{\omega_{\alpha}(1+\theta)^{p}} \int_{-\infty}^{\infty}|w(t)|^{p} e^{-t} \mathrm{~d} t
\end{aligned}
$$

and

$$
\int_{0}^{\infty} A_{p}\left(\mu|u(r)|^{q}\right) r^{\theta} \mathrm{d} r=\frac{1}{\theta+1} \int_{-\infty}^{\infty} A_{p}\left(\frac{\mu}{\mu_{\alpha, \theta}}|w(t)|^{q}\right) e^{-t} \mathrm{~d} t .
$$

Therefore, the proof of Theorem 1.4 is completed by showing that, for each $0<$ $\rho<1$, there exists a constant $C_{\rho}>0$ such that

$$
\int_{-\infty}^{\infty} A_{p}\left(\rho|w(t)|^{q}\right) e^{-t} \mathrm{~d} t \leq C_{\rho} \int_{-\infty}^{\infty}|w(t)|^{p} e^{-t} \mathrm{~d} t
$$

for all $C^{1}$ functions $w(t)$ satisfying (29) and such that $\int_{-\infty}^{\infty}\left|w^{\prime}(t)\right|^{p} \mathrm{~d} t=1$. To this end, consider

$$
A=\{t \in \mathbb{R}:|w(t)| \leq 1\} \quad \text { and } \quad B=\{t \in \mathbb{R}:|w(t)|>1\} .
$$

Clearly $A$ and $B$ are disjoint sets with $A \cup B=(-\infty, \infty)$, and by (29) we have $A \neq \emptyset$. Since $A_{p}(\zeta) \leq m_{p} \zeta^{\lfloor p\rfloor}$ when $0 \leq \zeta \leq 1$, for some $m_{p}>0$, we have

$$
\int_{A} A_{p}\left(\rho|w(t)|^{q}\right) e^{-t} \mathrm{~d} t \leq m_{p} \int_{-\infty}^{\infty}|w(t)|^{p} e^{-t} \mathrm{~d} t .
$$

By (29), the set $B$ has a lower bound and we can write $B=\bigcup_{i \in \mathbb{N}} I_{i}$ with $I_{i} \cap I_{j}=\emptyset$ if $i \neq j, I_{i}=\left(a_{i}, b_{i}\right), a_{i} \in A$ and $w\left(a_{i}\right)=1$. Thus, for each $i \in \mathbb{N}$, we obtain

$$
|w(t)| \leq 1+\int_{a_{i}}^{t}\left|w^{\prime}(s)\right| \mathrm{d} s \leq 1+\left(t-a_{i}\right)^{1 / q} \quad \text { for all } \quad t \in I_{i} .
$$

Note that given $\varepsilon>0$, there exists $C_{\varepsilon}$ such that $1+s^{1 / q} \leq\left((1+\varepsilon) s+C_{\varepsilon}\right)^{1 / q}$ for all $s \geq 0$, which together with (35) implies $|w(t)|^{q} \leq(1+\varepsilon)\left(t-a_{i}\right)+C_{\varepsilon}$ for all $t \in I_{i}$. Since $0<\rho<1$, taking $\varepsilon>0$ so that $\rho(1+\varepsilon)<1$, we can estimate

$$
\begin{aligned}
\int_{a_{i}}^{b_{i}} A_{p}\left(\rho|w(t)|^{q}\right) e^{-t} \mathrm{~d} t & \leq \int_{a_{i}}^{b_{i}} e^{\rho|w(t)|^{q}-t} \mathrm{~d} t \leq \int_{a_{i}}^{b_{i}} \exp \left\{(\rho(1+\varepsilon)-1)\left(t-a_{i}\right)+\rho C_{\varepsilon}-a_{i}\right\} \mathrm{d} t \\
& =\frac{e^{\rho C_{\varepsilon}}}{1-(\rho(1+\varepsilon))}\left(e^{-a_{i}}-e^{-b_{i}}\right) f\left(b_{i}-a_{i}\right),
\end{aligned}
$$

where $f(x)=\frac{e^{x}-e^{\rho(1+\varepsilon) x}}{e^{x}-1}, x \in \mathbb{R}$ and $f(\infty)=1$ if $b_{i}=\infty$. Since $|f(x)| \leq 1$ for all $x \in \mathbb{R}$, we have

$$
\int_{a_{i}}^{b_{i}} A_{p}\left(\rho|w(t)|^{q}\right) e^{-t} \mathrm{~d} t \leq \frac{e^{\rho C_{\varepsilon}}}{1-(\rho(1+\varepsilon))}\left(e^{-a_{i}}-e^{-b_{i}}\right) .
$$

Now, $|w|>1$ on $B$, which implies $\int_{a_{i}}^{b_{i}}|w(t)|^{p} e^{-t} \mathrm{~d} t \geq \int_{a_{i}}^{b_{i}} e^{-t} \mathrm{~d} t=\left(e^{-a_{i}}-e^{-b_{i}}\right)$. Hence, by (36) we get

$$
\sum_{i=1}^{\infty} \int_{a_{i}}^{b_{i}} A_{p}\left(\rho|w(t)|^{q}\right) e^{-t} \mathrm{~d} t \leq \frac{e^{\rho C_{\varepsilon}}}{1-(\rho(1+\varepsilon))} \sum_{i=1}^{\infty} \int_{a_{i}}^{b_{i}}|w(t)|^{p} e^{-t} \mathrm{~d} t .
$$


Therefore, by the monotone convergence theorem,

$\int_{B} A_{p}\left(\rho|w(t)|^{q}\right) e^{-t} \mathrm{~d} t \leq \frac{e^{\rho C_{\varepsilon}}}{1-(\rho(1+\varepsilon))} \int_{B}|w(t)|^{p} e^{-t} \mathrm{~d} t \leq \frac{e^{\rho C_{\varepsilon}}}{1-(\rho(1+\varepsilon))} \int_{-\infty}^{\infty}|w(t)|^{p} e^{-t} \mathrm{~d} t$.

Thus, combining (34) and (37), we have (33) for $C_{\rho} \geq m_{p}+e^{\rho C_{\varepsilon}} /(1-\rho(1+\varepsilon))$, which completes the proof.

\section{Proof of Theorem 1.5}

We consider Moser's sequence $w_{j}(t)$ given by $w_{j}(t)=0$ if $t \leq 0, w_{j}(t)=j^{-1 / p} t$ if $0 \leq t \leq j$ and $w_{j}(t)=j^{1 / q}$ if $t \geq j$. It is clear that

$$
\lim _{t \rightarrow-\infty} w_{j}(t)=0 \quad \text { and } \quad \int_{-\infty}^{\infty}\left|w_{j}^{\prime}(t)\right|^{p} \mathrm{~d} t=1 .
$$

We also have

$$
\int_{-\infty}^{\infty}\left|w_{j}(t)\right|^{p} e^{-t} \mathrm{~d} t \rightarrow 0 \quad \text { as } \quad j \rightarrow \infty
$$

Indeed,

$$
\int_{-\infty}^{\infty}\left|w_{j}(t)\right|^{p} e^{-t} \mathrm{~d} t=\frac{1}{j} \int_{0}^{j} t^{p} e^{-t} \mathrm{~d} t+j^{p-1} e^{-j} \rightarrow 0 \quad \text { as } \quad j \rightarrow \infty .
$$

A straightforward calculation yields

$$
\begin{aligned}
\int_{-\infty}^{\infty} A_{p}\left(\left|w_{j}(t)\right|^{q}\right) e^{-t} \mathrm{~d} t & =\int_{0}^{j} A_{p}\left(j\left(\frac{t}{j}\right)^{q}\right) e^{-t} \mathrm{~d} t+\int_{j}^{\infty} A_{p}(j) e^{-t} \mathrm{~d} t \\
& =\int_{0}^{j} \exp \left(j\left(\frac{t}{j}\right)^{q}-t\right) \mathrm{d} t-\sum_{m=0}^{\lfloor p\rfloor} \frac{1}{m !} j^{-\frac{m}{p-1}} \int_{0}^{j} t^{m q} e^{-t} \mathrm{~d} t \\
& +\left(e^{j}-\sum_{m=0}^{\lfloor p\rfloor} \frac{j^{m}}{m !}\right) e^{-j} \\
& \geq \int_{0}^{j} e^{-t} \mathrm{~d} t-\sum_{m=0}^{\lfloor p\rfloor} \frac{j^{-\frac{m}{p-1}}}{m !} \int_{0}^{j} t^{m q} e^{-t} \mathrm{~d} t+\left(e^{j}-\sum_{m=0}^{\lfloor p\rfloor} \frac{j^{m}}{m !}\right) e^{-j}
\end{aligned}
$$

Thus, for $j$ enough large,

$$
\int_{-\infty}^{\infty} A_{p}\left(\left|w_{j}(t)\right|^{q}\right) e^{-t} \mathrm{~d} t \geq \frac{1}{2}
$$

The proof of Theorem 1.5 follows by using an argument similar to the one in the proof of Theorem 1.4 combined with the estimates (38)-(40).

\section{ACKNOWLEDGEMENT}

The authors would like to thank the anonymous referee for valuable suggestions that improved the quality of the paper and for calling their attention to the paper [12], where the authors establish a weighted Trudinger-Moser inequality of the scaling invariant form as in our Theorems 1.4 and 1.5 for the more general framework when $\beta+2=n$, which corresponds to the particular case where $L$ is the $n$-Laplacian. 


\section{REFERENCES}

[1] Shinji Adachi and Kazunaga Tanaka, Trudinger type inequalities in $\mathbf{R}^{N}$ and their best exponents, Proc. Amer. Math. Soc. 128 (2000), no. 7, 2051-2057, DOI 10.1090/S0002-9939-9905180-1. MR 1646323 (2000m:46069)

[2] Robert A. Adams and John J. F. Fournier, Sobolev spaces, 2nd ed., Pure and Applied Mathematics (Amsterdam), vol. 140, Elsevier/Academic Press, Amsterdam, 2003. MR 2424078 (2009e:46025)

[3] George E. Andrews, Richard Askey, and Ranjan Roy, Special functions, Encyclopedia of Mathematics and its Applications, vol. 71, Cambridge University Press, Cambridge, 1999. MR:1688958 (2000g:33001)

[4] D. M. Cao, Nontrivial solution of semilinear elliptic equation with critical exponent in $\mathbf{R}^{2}$, Comm. Partial Differential Equations 17 (1992), no. 3-4, 407-435, DOI 10.1080/03605309208820848. MR1163431 (93f:35076)

[5] Lennart Carleson and Sun-Yung A. Chang, On the existence of an extremal function for an inequality of J. Moser (English, with French summary), Bull. Sci. Math. (2) 110 (1986), no. 2, 113-127. MR878016 (88f:46070)

[6] Philippe Clément, Djairo Guedes de Figueiredo, and Enzo Mitidieri, Quasilinear elliptic equations with critical exponents, Topol. Methods Nonlinear Anal. 7 (1996), no. 1, 133-170. MR.1422009 (97k:35072)

[7] D. G. de Figueiredo, J. V. Gonçalves, and O. H. Miyagaki, On a class of quasilinear elliptic problems involving critical exponents, Commun. Contemp. Math. 2 (2000), no. 1, 47-59, DOI 10.1142/S0219199700000049. MR1753138(2001j:35083)

[8] Djairo G. de Figueiredo, João Marcos do Ó, and Bernhard Ruf, On an inequality by N. Trudinger and J. Moser and related elliptic equations, Comm. Pure Appl. Math. 55 (2002), no. 2, 135-152, DOI 10.1002/cpa.10015. MR.1865413 (2002j:35104)

[9] Djairo G. de Figueiredo, João Marcos do Ó, and Bernhard Ruf, Elliptic equations and systems with critical Trudinger-Moser nonlinearities, Discrete Contin. Dyn. Syst. 30 (2011), no. 2, 455-476, DOI 10.3934/dcds.2011.30.455. MR2772124 (2011m:35083)

[10] João Marcos B. do Ó, $N$-Laplacian equations in $\mathbf{R}^{N}$ with critical growth, Abstr. Appl. Anal. 2 (1997), no. 3-4, 301-315, DOI 10.1155/S1085337597000419. MR1704875 (2001h:35045)

[11] Martin Flucher, Extremal functions for the Trudinger-Moser inequality in 2 dimensions, Comment. Math. Helv. 67 (1992), no. 3, 471-497, DOI 10.1007/BF02566514. MR.1171306 (93k:58073)

[12] M. Ishiwata, M. Nakamura, and H. Wadade, On the sharp constant for the weighted Trudinger-Moser type inequality of the scaling invariant form, Ann. Inst. H. Poincaré Anal. Non Linéaire 31 (2014), no. 2, 297-314. MR3181671

[13] Jon Jacobsen and Klaus Schmitt, Radial solutions of quasilinear elliptic differential equations, Handbook of differential equations, Elsevier/North-Holland, Amsterdam, 2004, pp. 359-435. MR2166492(2006k:34054)

[14] Jon Jacobsen and Klaus Schmitt, The Liouville-Bratu-Gelfand problem for radial operators, J. Differential Equations 184 (2002), no. 1, 283-298, DOI 10.1006/jdeq.2001.4151. MR.1929156 (2003g:34036)

[15] B. Opic and A. Kufner, Hardy-type inequalities, Pitman Research Notes in Mathematics Series, vol. 219, Longman Scientific \& Technical, Harlow, 1990. MR1069756 (92b:26028)

[16] Yu Xiang Li, Remarks on the extremal functions for the Moser-Trudinger inequality, Acta Math. Sin. (Engl. Ser.) 22 (2006), no. 2, 545-550, DOI 10.1007/s10114-005-0568-7. MR:2214376(2006m:35101)

[17] Kai-Ching Lin, Extremal functions for Moser's inequality, Trans. Amer. Math. Soc. 348 (1996), no. 7, 2663-2671, DOI 10.1090/S0002-9947-96-01541-3. MR.1333394 (96i:58043)

[18] Guozhen Lu and Yunyan Yang, Sharp constant and extremal function for the improved Moser-Trudinger inequality involving $L^{p}$ norm in two dimension, Discrete Contin. Dyn. Syst. 25 (2009), no. 3, 963-979, DOI 10.3934/dcds.2009.25.963. MR2533985 (2010m:35011)

[19] J. Moser, A sharp form of an inequality by N. Trudinger, Indiana Univ. Math. J. 20 (1970/71), 1077-1092. MR0301504 (46 \#662)

[20] H. L. Royden, Real analysis, 4th edition, Prentice Hall, New York, 2010. 
[21] Michael Struwe, Critical points of embeddings of $H_{0}^{1, n}$ into Orlicz spaces (English, with French summary), Ann. Inst. H. Poincaré Anal. Non Linéaire 5 (1988), no. 5, 425-464. MR.970849 (90c:35084)

[22] Neil S. Trudinger, On imbeddings into Orlicz spaces and some applications, J. Math. Mech. 17 (1967), 473-483. MR0216286 (35 \#7121)

Department of Mathematics, Federal University of Pernambuco, 50740-560 Recife, PE, BRAZIL

E-mail address: oliveira@dmat.ufpe.br

Department of Mathematics, Federal University of Paraíba, 58051-900 Joũo Pessoa, PB, BRAZIL

E-mail address: jmbo@pq.cnpq.br 